\title{
Atomic Layer-Deposited Al-Doped ZnO Thin Films for Display Applications
}

\author{
Dimitre Dimitrov ${ }^{1,2, *}$, Che-Liang Tsai ${ }^{3}$, Stefan Petrov ${ }^{4}$, Vera Marinova ${ }^{2,4, *}$, \\ Dimitrina Petrova ${ }^{2,5}$, Blagovest Napoleonov ${ }^{2}$, Blagoy Blagoev ${ }^{1}$, Velichka Strijkova ${ }^{2}$, \\ Ken Yuh Hsu ${ }^{3}$ and Shiuan Huei Lin ${ }^{4}$ \\ 1 Institute of Solid State Physics, Bulgarian Academy of Sciences, 1784 Sofia, Bulgaria; blago_sb@yahoo.com \\ 2 Institute of Optical Materials and Technologies, Bulgarian Academy of Sciences, 1113 Sofia, Bulgaria; \\ d_kerina@abv.bg (D.P.); blgv@abv.bg (B.N.); vily@iomt.bas.bg (V.S.) \\ 3 Department of Photonics, National Chiao Tung University, Hsinchu 30010, Taiwan; \\ Patrick.Tsai@crystalvue.com.tw (C.-L.T.); ken@cc.nctu.edu.tw (K.Y.H.) \\ 4 Department of Electrophysics, National Chiao Tung University, Hsinchu 30010, Taiwan; \\ st5pob@gmail.com (S.P.); lin@cc.nctu.edu.tw (S.H.L.) \\ 5 Faculty of Engineering, South-West University “Neofit Rilski”, 2700 Blagoevgrad, Bulgaria \\ * Correspondence: dzdimitrov@issp.bas.bg (D.D.); vmarinova@iomt.bas.bg (V.M.)
}

Received: 30 April 2020; Accepted: 25 May 2020; Published: 31 May 2020

\begin{abstract}
The integration of high uniformity, conformal and compact transparent conductive layers into next generation indium tin oxide (ITO)-free optoelectronics, including wearable and bendable structures, is a huge challenge. In this study, we demonstrate the transparent and conductive functionality of aluminum-doped zinc oxide (AZO) thin films deposited on glass as well as on polyethylene terephthalate (PET) flexible substrates by using an atomic layer deposition (ALD) technique. AZO thin films possess high optical transmittance at visible and near-infrared spectral range and electrical properties competitive to commercial ITO layers. AZO layers deposited on flexible PET substrates demonstrate stable sheet resistance over 1000 bending cycles. Based on the performed optical and electrical characterizations, several applications of ALD AZO as transparent conductive layers are shown-AZO/glass-supported liquid crystal (LC) display and AZO/PET-based flexible polymer-dispersed liquid crystal (PDLC) devices.
\end{abstract}

Keywords: Al-doped ZnO; ALD technique; transparent conductive layers; LC display; flexible PDLC devices

\section{Introduction}

Transparent conducting materials (TCMs) possess simultaneously high electrical conductivity and optical transparency (i.e., effective transmission of light in the visible spectrum), with these particular characteristics being achieved on processing the materials as thin films on transparent substrates. This specific combination of properties makes TCMs very interesting both from a fundamental viewpoint and for a large variety of applications. The principal factor that has stimulated the research on TCM syntheses and their processing is definitely the development of optoelectronic materials and devices in which the principles of actuation involve an application of electric current or voltage to control the emission or passage of light, the most notable examples being display devices $[1,2]$. Moreover, other applications that require TCM, such as smart windows (based on electrochromic or polymer-dispersed liquid crystalline materials) and photovoltaic systems, have attained enormous relevance in the present context of environmentally important energy efficiency and clean energies, further prompting the scientific and technical developments in TCMs, as evidenced in the intensive and continuing research in this field [3]. 
The most studied and practically used TCMs are oxides, referred to as transparent conducting oxides (TCOs). TCOs are usually made of high band gap $(>3 \mathrm{eV})$ oxides which are intrinsically or extrinsically doped to reach a very low resistivity $\left(\sim 10^{-4} \Omega \cdot \mathrm{cm}\right)$. The basic TCO materials include indium oxide $\left(\mathrm{In}_{2} \mathrm{O}_{3}\right)$, tin oxide $\left(\mathrm{SnO}_{2}\right)$, and zinc oxide $(\mathrm{ZnO})$. They can be degenerately n-type doped with tin $\left(\mathrm{In}_{2} \mathrm{O}_{3}: \mathrm{Sn}\right.$, also known as ITO), fluorine $\left(\mathrm{SnO}_{2}: \mathrm{F}\right)$ or $\mathrm{Al}(\mathrm{ZnO}: \mathrm{Al})$, as popular examples. The usage of TCO films depends on the application. In liquid crystal displays (LCDs), light-emitting diodes (LEDs), or transparent displays, these films are used as electrodes, while they are used as touch sensors for resistive and capacitive touch panels. Transparent electrodes are essential in high-impact technological areas such as photovoltaics, flat panel displays and touch screens, as well as in emerging areas such as smart sensors or organic electronics (organic light-emitting devices (OLEDs), organic photovoltaics) as well as transparent field-effect transistors (FETs) [4]. The most relevant properties for all these applications are their high conductivity combined with high transparency in the visible spectral region. Furthermore, key TCO performance factors, depending on the particular application, are their high chemical and thermal stability or the possibility of tuning their work function. Especially important for flexible electronics are the mechanical properties, high stretchability and bendability, and low-contact resistance with organic materials. Transparent conducting films are estimated to reach a market of value of US\$ 8.46 billion by 2026 [5]; intensive research is therefore important to discover superior materials, new substrates, and new ways to enhance light transmission, to increase the electric conductivity, to add flexibility, and to decrease costs.

ITO is the most widely used material for the fabrication of TCOs; however, ITO has several critical shortcomings such as generally high processing cost and unsuitability for flexible devices. In particular, for large-area touch screens, the resistivity is too high for the rapid touch sensing response; in addition, ITO is brittle and therefore inadequate for applications in flexible electronics such as flexible touch screen displays and solar cells. Aluminum-doped zinc oxide (AZO) is an affordable, non-toxic and robust transparent conductive oxide (TCO) [6]. AZO films have high transmission in the visible region and useable transmission to IR wavelengths as long as $\sim 12 \mu \mathrm{m}$. In contrast, the more commonly known TCO, ITO, reflects IR at wavelengths longer than $\sim 2 \mu \mathrm{m}$. IR transmission is very important because increasing the long-wavelength response is an approach to enhance the efficiency of some solar devices [7]. The higher stability of AZO in reducing atmospheres may also be an advantage for future applications [8]. A substantial cost saving is possible with AZO materials compared to ITO and other TCOs. Due to these practical advantages, AZO films are considered as ideal replacements for ITO films in applications such as transparent electrodes for solar cells, flat panel displays, LCD electrodes, touch panel transparent contacts and IR windows [9-11]. AZO thin films can be deposited by several techniques such as sol-gel [12], chemical spray [13], thermal evaporation [14], pulsed laser deposition [15], DC and RF magnetron sputtering [16], reactive mid-frequency magnetron sputtering using dual magnetrons [17] and atomic layer deposition (ALD) [18]. The desired properties of a good TCO—transparency, conductivity, and surface texture-depend significantly on the preparation technique and the growth parameters.

ALD is a growth technique that has recently become very popular since it provides uniform and conformal coverage and control of the thin film by atomic layer precision [19]. Although the growth rate of the ALD system is relatively low, the uniformity, conformality and the compactness of the film cross-section achieved from the ALD technique are superior to those from other techniques [20].

In this work, we demonstrate the application of AZO thin films as a substitute for ITO electrodes in LC and PDLC display devices. Using the ALD technique, AZO films are deposited on glass and PET substrates and their structural, optical and electric properties are measured and discussed. AZO layers on PET substrates show good flexing properties, as evidenced by their stable sheet resistance over 1000 bending cycles.

The article is structured as follows: we first report on the ALD deposition of AZO films, followed by brief characterizations of the layers, including structural, optical and electrical properties. The next step includes the measurement of the bending ability of the AZO layer deposited on a flexible PET substrate. 
In the final section, on the basis of the above characteristics, we present two applications of AZO as a transparent and conductive layer, respectively, in (i) a liquid crystal (LC) display using AZO/glass and (ii) flexible polymer-dispersed liquid crystal (PDLC) devices using AZO/PET. The obtained results show the great potential of AZO for integration into next-generation ITO-free flexible and stretchable devices.

\section{Materials and Methods}

Al-doped ZnO films were deposited using an ALD Beneq TFS-200 system (Espoo, Finland). Trimethylaluminum (TMA) and diethylzinc (DEZ) were purchased from Strem Chemicals, Inc. (Bischheim, France), and used as received. These precursors and distilled water were held at room temperature in stainless steel containers for all depositions. Purge and line flows used pure $\mathrm{N}_{2}$ (600 sccm for TMA, DEZ, and water). The precursors' pulse durations were the same for DEZ, TMA, and $\mathrm{H}_{2} \mathrm{O}-200 \mathrm{~ms}$, while the purging time after each precursor was $2 \mathrm{~s}$, for all deposition runs. In all depositions, the TMA pulse was introduced after a DEZ pulse/purge cycle, in order to minimize the impact of the TMA pulse on the growth rate [21]. The Al-doping of $\mathrm{ZnO}$ was controlled by varying the number of DEZ/ $\mathrm{H}_{2} \mathrm{O}$ and TMA $/ \mathrm{H}_{2} \mathrm{O}$ pulses [22]. In a typical deposition procedure, after each 24 cycles of DEZ/ $\mathrm{H}_{2} \mathrm{O}$, a cycle of TMA/ $\mathrm{H}_{2} \mathrm{O}$ was applied consisting one so-called supercycle. The desired film thickness was controlled by the number of supercycles. The depositions were performed with the substrate holder temperature of $200{ }^{\circ} \mathrm{C}$ for AZO/glass and $100^{\circ} \mathrm{C}$ for AZO/PET. The structural, optical and electrical properties of ALD Al-doped $\mathrm{ZnO}$ films were measured by using Atomic Force Microscopy (AFM), Scanning Electron Microscopy (SEM), spectrophotometry and a four-point probe method.

Additionally, in the case of AZO deposition on PET substrates, a buffer film of amorphous $\mathrm{Al}_{2} \mathrm{O}_{3}$ (ALO) was deposited in situ at the same temperature of $100^{\circ} \mathrm{C}$. The buffer film prevents the diffusion of the next precursors in the pores of the PET substrate. For ALO buffer deposition, the same precursors ( TMA $/ \mathrm{H}_{2} \mathrm{O}$ ) were used with the same pulse durations and purging times and 88 repetitions of the TMA/ $\mathrm{H}_{2} \mathrm{O}$ cycle.

The AZO film thickness was determined by ellipsometric measurements using a Woollam M2000D rotating compensator spectroscopic ellipsometer (Lincoln, MI, USA) with a wavelength range from 193 to $1000 \mathrm{~nm}$ in reflection mode. For AZO/glass, the thickness was $206 \mathrm{~nm}$ and for AZO/PET the thickness was approximately $100 \mathrm{~nm}$. The $\mathrm{Al}_{2} \mathrm{O}_{3}$ buffer film deposited on PET substrates as barrier to prevent diethylzinc diffusion into the polymer substrate [23] was approximately 15-nm thick.

The AZO films' surface morphology was analyzed using Atomic Force Microscopy (AFM), MFP-3D, Asylum Research, Oxford Instruments (Abingdon, UK) and Scanning Electron Microscopy (SEM), JEOL (Tokyo, Japan).

The optical transmittance spectra of AZO films deposited on glass and PET in the wavelength range of 200 to $1600 \mathrm{~nm}$ were measured at room temperature using an ultraviolet-visible-near-infrared (UV-VIS-NIR) spectrophotometer Cary 5E (Palo Alto, CA, USA). The same measurements were performed on reference glass, PET and ITO/glass substrates for comparison.

The sheet resistance was measured using a four-point probe technique. A computerized home-built bending setup, with an ESP301 control platform (Newport, Irvine, CA, USA) with the function to stimulate bending at different radii, was used for the bending tests of flexible samples. The sheet resistance was tested in the interval of up to 1000 bending cycles.

Several AZO films deposited on glass and PET were selected for the LC and PDLC device assembly. The LC and PDLC device fabrication procedure is described in detail in Section 3.2: Applications. The electro-optical characteristics of fabricated LC and PDLC devices were measured by positioning the assembled cell in the optical setups, also described in detail in the same section.

\section{Results and Discussion}

For an application as a transparent conductive layer, the high optical transmission and high electrical conductivity of the AZO layers are the most essential properties. These properties also 
depend on the film's elemental composition (doping concentration), deposition conditions/method and the interface with the substrate.

\subsection{AZO Layers Characterisations}

\subsubsection{Structural Analysis}

The surface morphologies of the samples were analyzed by using the AFM method with a scanning area of $40 \times 40 \mu^{2}$, as well as SEM. AFM and SEM images for the surface morphologies of AZO films on glass and PET (before and after bending) are shown in Figure 1a-f. The surface of the films on glass was reasonably smooth, with a roughness of $<3 \mathrm{~nm}$. Grain growth was dense with highly homogenous distribution, as clearly seen in the 3-D AFM image of the surface. It is well known that the grain size, the distribution and the surface roughness are influenced by the sample thickness [16]. The measured root mean square (RMS) roughness of AZO/glass was about $2.3 \mathrm{~nm}$. As seen from the SEM, the as-grown AZO films on both substrates-glass and PET are relatively smooth, with a uniform distribution of slightly elongated grains. The average grain size of AZO films is approximately the same, around $20 \mathrm{~nm}$. The RMS roughness of AZO/PET and SEM morphology before and after bending are discussed in Section 3.1.4.
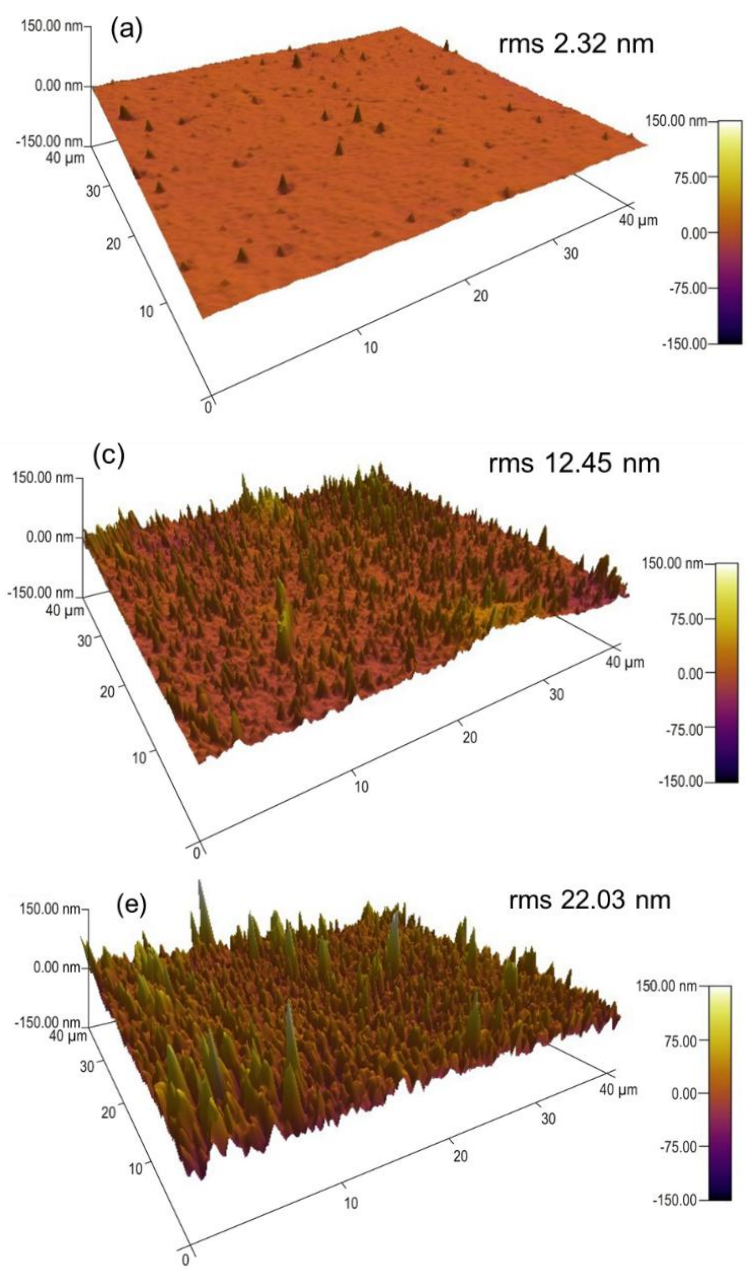
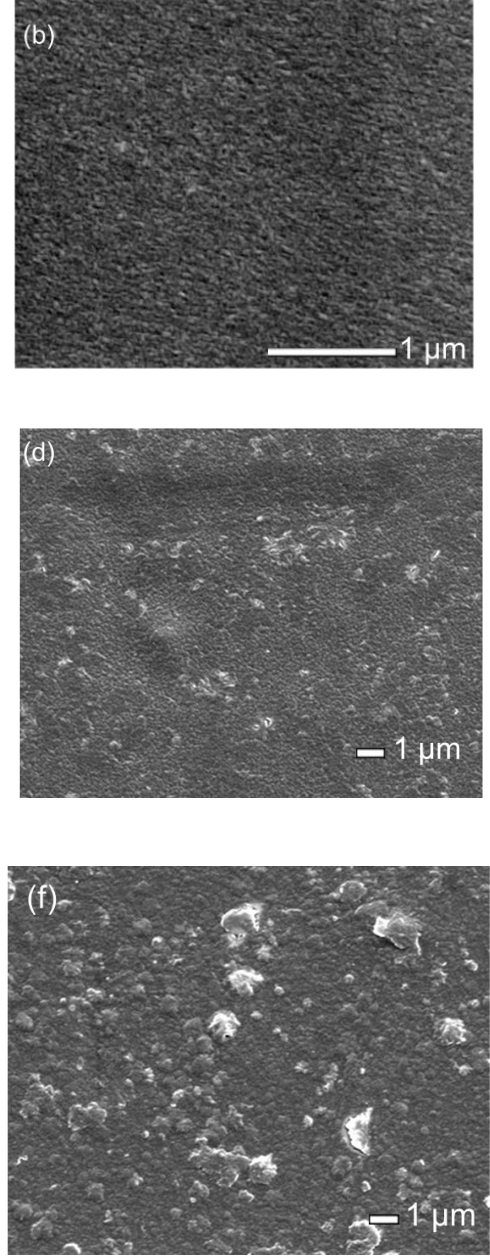

Figure 1. Atomic Force Microscopy (AFM) and SEM analysis of aluminum-doped zinc oxide (AZO) layers deposited on $(\mathbf{a}, \mathbf{b}) \mathrm{AZO} /$ glass $(\mathbf{c}, \mathbf{d}) \mathrm{AZO} /$ polyethylene terephthalate (PET) (before bending test) and (e,f) AZO/PET (after bending 1000 times, discussed in 3.1.4.). 


\subsubsection{Optical Properties}

The optical transmittance spectra of AZO films deposited on glass and PET are shown in Figure 2. The spectra of glass and PET blank substrates are also included for reference as well as commercially available ITO/glass substrate. As seen in comparison to the blank PET substrate, the absorption edges of AZO films on glass and PET are shifted to the longer wavelengths and follow a similar transmittance behavior (around $80 \%$ transmittance in visible and near-infrared regions).

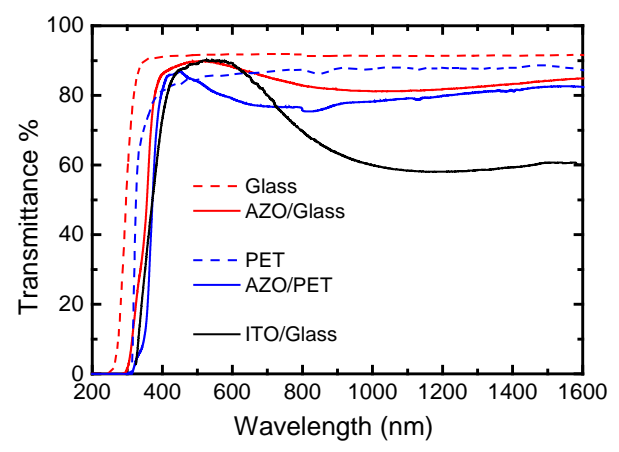

Figure 2. Transmittance spectra of AZO/glass and AZO/PET. Blank glass and PET spectra are also shown for reference, as well as commercial indium tin oxide (ITO)/glass.

\subsubsection{Electrical Properties}

Next, the electrical properties were assessed. Room temperature currents versus voltage (I-V) characteristics of AZO thin films on glass and PET as well as ITO/glass are shown in Figure 3a-c. All performed current-voltage measurements show Ohmic behavior. As a device-relevant parameter, sheet resistance $\left(R_{S}\right)=1 / \sigma d$, where $\sigma$ is the electrical conductivity of the material and $d$ is the thickness of the electrode, is used for a comparison of the transparent electrodes rather than conductivity, which is an intrinsic material property. The calculated sheet resistances are also shown in the figures.

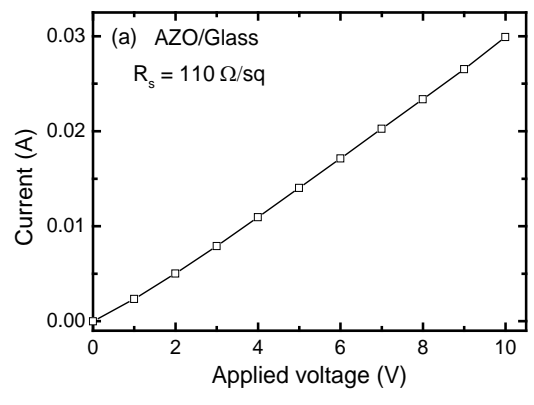

(a)

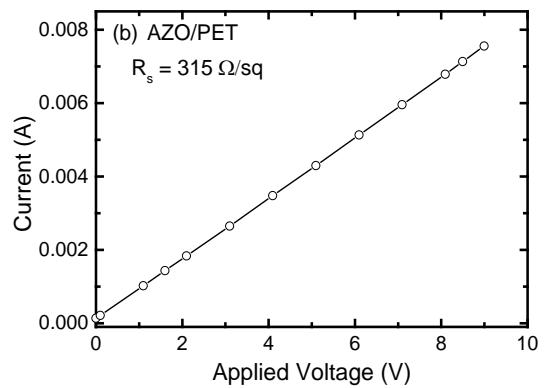

(b)

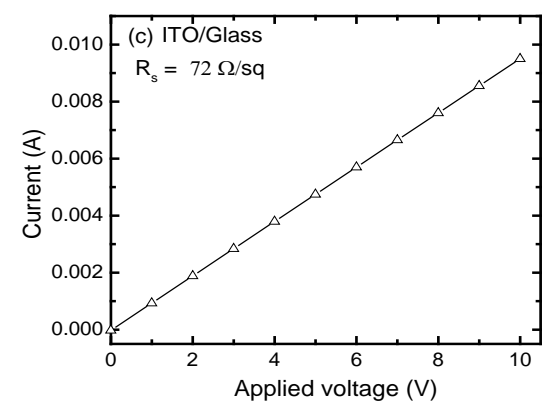

(c)

Figure 3. Current-voltage characteristics of AZO thin films deposited on (a) glass and (b) PET, as well a comparison with (c) commercial ITO/glass, are shown for reference. 


\subsubsection{Bending Ability Test of AZO/PET Flexible Substrates}

The bending ability test has been checked for flexible AZO/PET substrates under tensile strain. Generally, the sheet resistance $\left(R_{\mathrm{s}}\right)$ value depends on the film composition, morphology, thickness and the substrate material. After continuous bending up to 1000 times (radius of $10 \mathrm{~mm}$ ), the sheet resistance of AZO/PET increased only slightly (see Figure 4). The RMS roughness of blank PET substrate was $6.26 \mathrm{~nm}$. AZO/PET RMS was measured to be around $12 \mathrm{~nm}$ and it is increasing to $22 \mathrm{~nm}$ after 1000 bending cycles. During the bending cycles, damage in the AZO/PET develops, most probably in the form of extended defects that collapse as micro-cracks (as evidenced by SEM image in Figure 1f), capable of interrupting electrical paths on subsequent bending. Bending in-out cycles open and close these micro-cracks, and with the accumulation of the bending cycles an effective breaking of a slightly increasing number of conductive paths occurred, thus influencing the measured resistance and appearing as increased sheet resistance. These results are confirmed further by the increased roughness shown in the AFM image of the same layer, performed after 1000 bending cycles (Figure 1c).

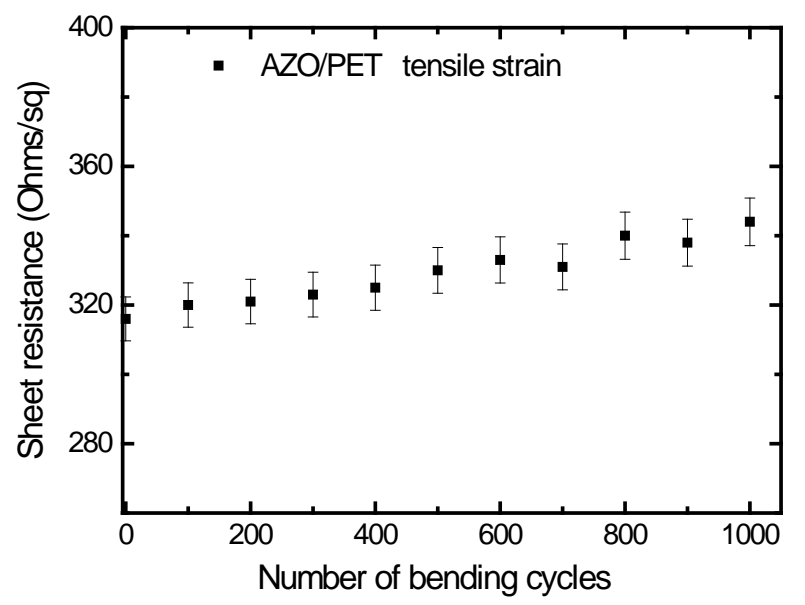

Figure 4. Sheet resistance dependence of the number of bending cycles for AZO layer on PET substrate.

\subsection{Applications of AZO Transparent Conductive Layer for Display Applications}

\subsubsection{AZO as Transparent Conductive Layer in Rigid LC Display}

Several AZO/glass samples were selected for transparent electrodes in the LC cell assembly, hereafter called an AZO-based LC rigid display. First, a Polyvinyl Alcohol (PVA) layer was spin-coated, baked at $90^{\circ} \mathrm{C}$ for $30 \mathrm{~min}$ and post baked at $120^{\circ} \mathrm{C}$ for $30 \mathrm{~min}$ on $\mathrm{AZO} /$ glass samples. Next, the PVA-coated AZO/glass substrates were mechanically rubbed for LC molecule alignment. We used nematic LC (NLC type E7, Merck, Kenilworth, NJ, USA). The cell was arranged by gluing the two rubbed layers in an anti-parallel configuration with the alignment directions facing each other. A 12- $\mu \mathrm{m}$ Mylar spacer was used to keep the same thicknesses in the fabricated cells. The liquid crystal was injected via the capillary method into the empty cell. Finally, the arranged cell was sealed with ultraviolet (UV) glue and exposed with UV light to stabilize it. Copper tape wires were used for electrical connections (see Figure 5a,b). In addition, a reference LC cell using ITO/glass was prepared following the same fabrication procedure and thickness for comparison.

For electro-optical modulation characteristic measurements, the LC cells were placed between a pair of crossed polarizers. The incident beam was polarized at the angle of $45^{\circ}$ with respect to the nematic director (a dimensionless unit vector $n$, which represents the direction of the preferred orientation of LC molecules). An alternating voltage ( $f=1 \mathrm{kHz})$ with varying amplitude was applied across to the LC cell to orient the LC director. A helium-neon laser (He-Ne) emitting $\lambda=633 \mathrm{~nm}$ was used to probe the changes in the transmitted intensity of LC devices under different amplitudes of driving voltage. The power of the transmitted beam was monitored by a power meter. The light 
transmission was measured as a function of the applied root mean square (RMS) alternating current (AC) voltage with a 1-kHz frequency. The transmittance changes were detected by positioning a photodetector behind the device (Figure $5 \mathrm{~d}$ ).

a) AZO/Glass LC cell

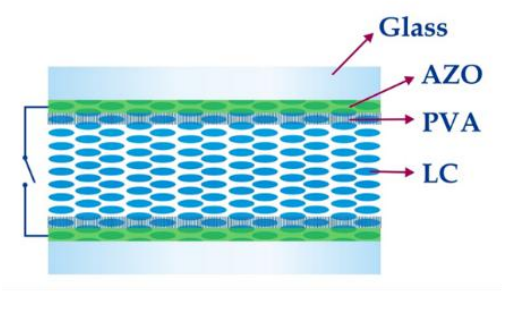

b) ITO/Glass LC cell

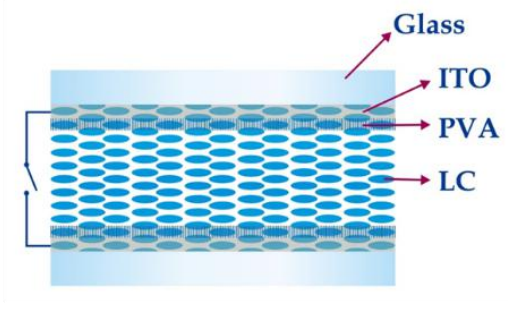

c) AZO/PET PDLC cell

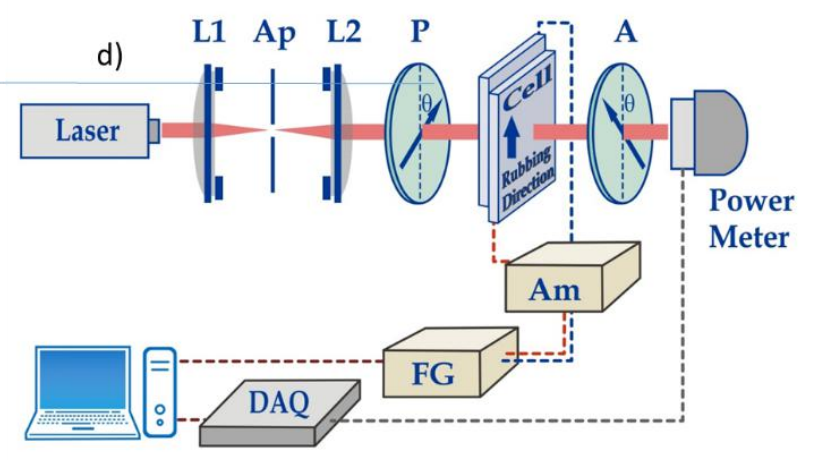

Figure 5. Schematic diagrams of: (a) AZO/glass LC cell; (b) ITO/glass LC cell and (c) AZO/PET polymer-dispersed liquid crystal (PDLC) cell and (d) experimental setup to measure the electro-optical modulation behavior. Legend: lens (L), aperture (Ap), polarizer (P), analyzer (A), amplifier (Am), function generator (FG), data acquisition card (DAQ). Note: for PDLC cell measurements, the P and A are removed from the setup.

The basic operation principles of LC displays rely on the electro-optically controlled birefringence of the LC molecules [24,25]. Upon application of an electric field, since the LC molecules have a different polarizability along their long and short axis, an induced dipole moment arises and all the molecules start to reorient towards the direction of the applied field. Because of the LC's anisotropy, the nematic LC layer acts as the birefringent material, characterized by different refractive indices for a beam polarized along the long or short molecular axis.

Figure $6 \mathrm{a}, \mathrm{b}$ shows the transmitted light intensity dependence of the applied voltage for an LC cell using AZO as transparent electrode. A reference cell, assembled using a commercial ITO electrode is shown for comparison. The transmittance-voltage behavior follows the typical sinusoidal function of the amplitude of applied voltage, governed by the electrical response of the liquid crystal molecules, as expressed by [25].

$$
T=\frac{1}{2} \sin ^{2} \frac{\Gamma}{2}=1 / 2 \sin ^{2}\left[\frac{\pi d\left(n_{\mathrm{e}}-n_{0}\right)}{\lambda}\right]
$$

where $\Gamma=\frac{2 \pi}{\lambda}\left(n_{\mathrm{e}}-n_{0}\right) d$ is the phase retardation due to the birefringence $\Delta n\left(\Delta n=n_{\mathrm{e}}-n_{0}\right)$ modulation, $\lambda$-the wavelength, $d$-the thickness of the LC layer and $n_{\mathrm{e}}$ and $n_{0}$ are the refractive indices for extraordinary and ordinary waves, respectively.

As shown in Figure 6a, the transmitted intensity follows a series of maxima and minima (so-called Fréedericksz transition), which correspond to the phase retardation, as presented in Figure 6b.

The low sheet resistance of the AZO layer has a large impact on the modulation characteristics of assembled LC cells. As seen from Figure 6a, the modulation behavior of the LC cell using AZO contacts is very similar and competitive with the LC cell using ITO. The results are also supported by 
the calculated Haacke figure of merit (FOM) factor of performance, expressed by the ratio between the optical transmittance $\left(T_{\mathrm{av}}\right)$ and the sheet resistance $\left(R_{\mathrm{s}}\right)$ values [26]:

$$
\mathrm{FOM}=\frac{T_{a v} 10}{R_{S}}
$$

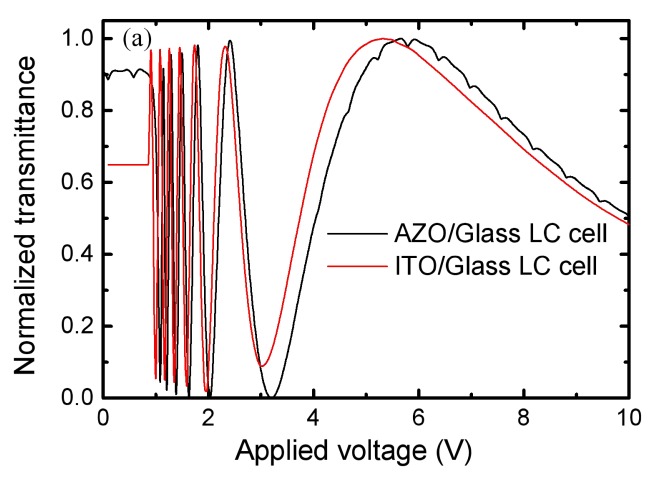

(a)

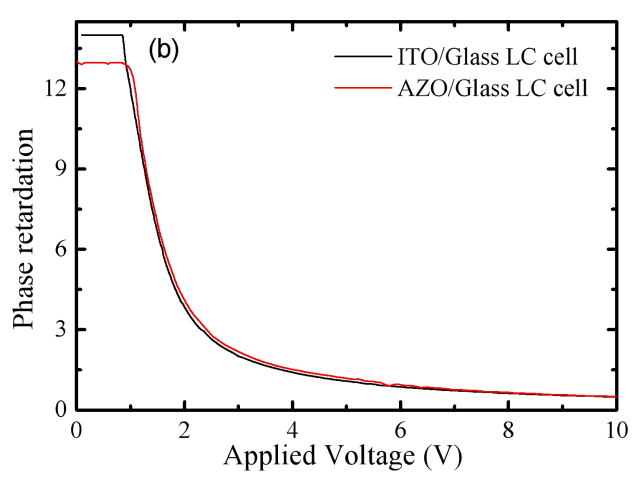

(b)

Figure 6. (a) Transmittance-voltage characteristics of AZO/glass and ITO/glass (reference) LC cells and (b) phase retardation for both LC cells.

Using the transmittance data (Figure 2) and measured sheet resistance values of AZO/glass, we obtained FOM $_{(\mathrm{AZO})}=1.05 \times 10^{-3} \Omega^{-1}$. Similar values have been calculated for (commercially available) ITO/glass, FOM $\left(\right.$ ITO) $=2.52 \times 10^{-3} \Omega^{-1}$. These values are in good confirmation with those previously reported $[27,28]$ and demonstrate the high potential of AZO films as transparent electrodes for ITO-free LCD devices (Table 1).

Table 1. Haacke figures of merit (FOM).

\begin{tabular}{cccc}
\hline LC Cell Type & $\boldsymbol{T}_{\text {av }}(\mathbf{\%})$ & $\boldsymbol{R}_{\mathbf{s}}(\boldsymbol{\Omega} / \mathbf{s q})$ & FOM $\left(\boldsymbol{\Omega}^{-\mathbf{1}}\right)$ \\
\hline AZO/Glass & 80.6 & 110 & $1.05 \times 10^{-3}$ \\
ITO/Glass & 84.3 & 72 & $2.52 \times 10^{-3}$ \\
\hline
\end{tabular}

\subsubsection{AZO as Transparent Conductive Layer in Flexible Display (Smart Structures)}

PDLC-based structures have attracted increasing attention for applications as outdoor displays, switchable privacy glasses, energy saving windows, light shutters, projection displays, and so on [29,30]. Here, the PDLC film was prepared using a polymerization-induced phase separation method, based on the phase separation of the LC (E7, Merck, $n_{0}=1.521$ and $\left.n_{\mathrm{e}}=1.72\right)$ and photo-curable adhesive polymer matrix (NOA65, Norland, Cranbury, NJ, USA, $n=1.524$ ) with a 3:7 weight ratio. During the fabrication of our flexible PDLC structures, supported by AZO/PET substrates, we perform the following steps: first, an empty cell was prepared by gluing two AZO/PET substrates with 12- $\mu \mathrm{m}$ Mylar spacers between them. Then, the LC/monomer mixture was injected into the empty cell and, finally, the cell was exposed with ultraviolet light $\left((\lambda=365 \mathrm{~nm})\right.$ with an intensity of $60 \mathrm{~mW} / \mathrm{cm}^{2}$ for $15 \mathrm{~min}$ ) to polymerize NOA65. As a result, using the polymerization-induced phase separation method, randomly dispersed liquid crystal droplets (usually in micrometer size) are formed within a transparent polymer matrix. Figure 5c shows the schematic structure of the AZO/PET PDLC flexible device.

\subsubsection{Electro-Optical Characteristics and Bending Test Ability}

In general, PDLC can be switched between a light-scattering to a transparent state by applying an external electric field. The effect results from a mismatch or match of refractive indices between the LC 
molecules and the polymer matrix and is due to the LC birefringence and the ability of an applied voltage to re-orient the LC molecules inside the droplets in order to match the LC's refractive index to that of the polymer matrix. The electro-optical characteristics were measured by an optical setup, shown in Figure 5d. In these measurements, the polarizer and analyzer were removed from the setup, since the polymer defines the polarization state of the PDLC film.

The typical transmittance dependence of the assembled AZO/PET PDLC device as a function of the applied voltage is shown in Figure 7a. Without an applied voltage, the LC molecules are randomly oriented in the droplets, which causes light scattering when the light passes through the structure. As a result, the "scattering" state appears. When the voltage is applied, the electric field aligns the LC's nematic director to the direction of the electric field, allowing light to pass through the droplets. As a result, a "transparent" state appears. Thus, the intensity of the transmitted light through the PDLC structure can be controlled by the application of an external voltage.
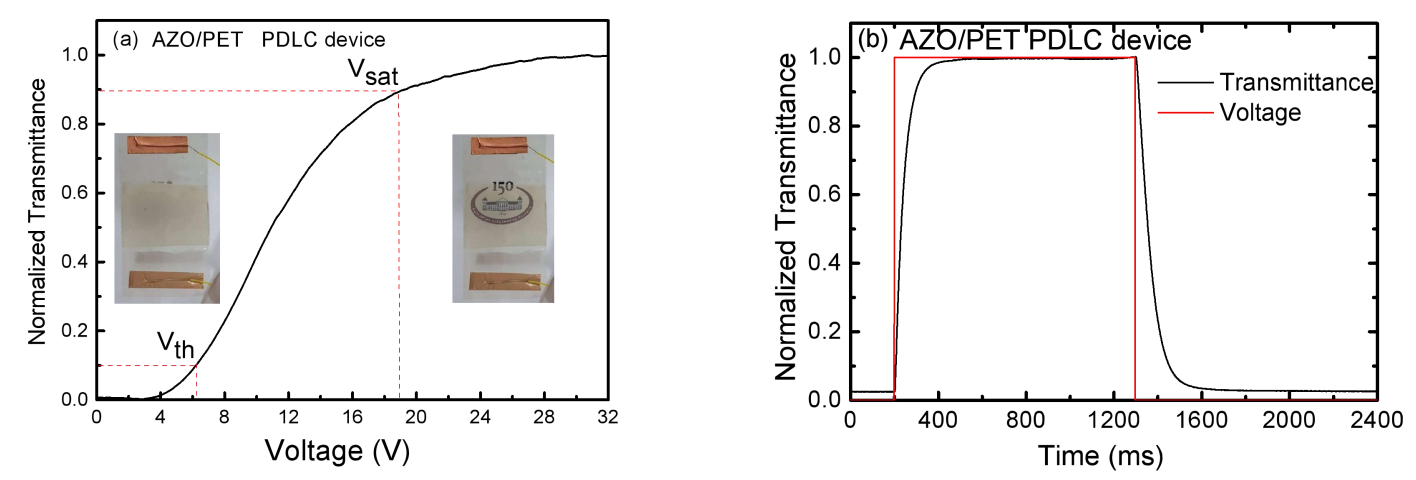

Figure 7. (a) Voltage-transmittance behavior of AZO/PET PDLC device and (b) the response time.

For the AZO/PET device shown in Figure 7a, we defined (i) the threshold voltage $V_{\text {th }}$ as a value of the applied voltage necessary to reach $10 \%$ of the maximum transmittance, e.g., to "turn on" the PDLC cell (measured $V_{\text {th }} \sim 6.1 \mathrm{~V}$ ) and (ii) the saturation voltage $V_{\text {sat }}$, defined as the value of applied voltage required to reach $90 \%$ of maximum transmittance $\mathrm{T}\left(V_{\text {sat }} \sim 18 \mathrm{~V}\right)$. As seen, at the saturation state, the PDLC structure becomes transparent (the "BAS LOGO" image pattern become clear).

In addition, the response time of the assembled PDLC device for switching between "off" and "on" states was measured and is presented in Figure $7 \mathrm{~b}$. The measured response time and the fall time values were $\sim 68$ and $\sim 88 \mathrm{~ms}$, respectively. These values are very similar to the reported values for PDLC devices using other transparent contacts [31,32].

To sum up, the implementation of AZO layers as transparent conductive electrodes in LC and PDLC devices requires the synthesis of high uniformity, conformal and compact layers by an appropriate process such as ALD. The AZO films obtained by ALD technique enable low sheet resistance and high optical transparency through the use as transparent electrodes on the top surfaces of both the selected rigid and flexible substrates.

\section{Conclusions}

In summary, we studied the structural and optical properties and the sheet resistance ability of AZO layers deposited by the ALD technique onto glass and PET substrates. LC devices with transparent AZO electrodes were successfully fabricated, and the measured driving voltage and response time values show the great potential of AZO for integration as functional transparent conducting material. We expect that the above demonstrations will be relevant for further applications in high-end optoelectronics.

Author Contributions: Conceptualization, V.M., S.H.L., and K.Y.H.; methodology D.D., B.B., S.P., and D.P.; software, S.P., C.-L.T., V.S., and B.N.; formal analysis, V.M., S.H.L., and K.Y.H.; resources D.D., V.M., and S.H.L.; writing-original draft preparation, D.D., and V.M.; writing—review and editing D.D., C.-L.T., V.M., B.B., S.P., 
D.P., V.S., B.N., S.H.L., and K.Y.H.; visualization, D.D., C.-L.T., V.M., S.P., D.P. and V.S.; supervision, V.M., S.H.L., and K.Y.H.; funding acquisition D.D., V.M., and S.H.L. All authors have read and agreed to the published version of the manuscript.

Funding: This research was funded by the Bulgarian Science Fund under the Grant No. KП-06-H-28/8 and the Ministry of Science and Technology (MOST), Taiwan, under the Grant No.: MOST 107-2221-E-009-120-MY3 and MOST 109-2927-I-009-507. We are grateful for the support from the Higher Education Sprout Project of the National Chiao Tung University and Ministry of Education (MOE), Hsinchu, Taiwan. Research equipment from the distributed research infrastructure INFRAMAT (part of the Bulgarian National roadmap for research infrastructures), supported by the Bulgarian Ministry of Education and Science under contract D01-284/17.12.2019, was used for AFM measurements.

Acknowledgments: The authors would like to thank P. Terziyska from the ISSP, BAS, Bulgaria for determining the AZO layer's thickness and to Marina Vasileva from the IOMT, BAS, Bulgaria for the measurement of the optical spectra. The authors gratefully acknowledge the financial support provided by the Bulgarian Science Fund under the grant number KП-06-H-28/8 and the Ministry of Science and Technology (MOST), Taiwan, under the grant numbers MOST 107-2221-E-009-120-MY3 and MOST 109-2927-I-009-507 (Bulgarian-Taiwanese PPP exchange program). We are grateful for the support from the Higher Education Sprout Project of the National Chiao Tung University and Ministry of Education (MOE), Taiwan. Research equipment from the distributed research infrastructure INFRAMAT (part of the Bulgarian National roadmap for research infrastructures), supported by the Bulgarian Ministry of Education and Science under contract D01-284/17.12.2019, was used for AFM measurements.

Conflicts of Interest: The authors declare no conflict of interest.

\section{References}

1. Ellmer, K. Past achievements and future challenges in the development of optically transparent electrodes. Nat. Photonics 2012, 6, 809-817. [CrossRef]

2. Gordon, R.G. Criteria for choosing transparent conductors. MRS Bull. 2000, 25, 52-57. [CrossRef]

3. Morales-Masis, M.; De Wolf, S.; Woods-Robinson, R.; Ager, J.W.; Ballif, C. Transparent electrodes for efficient optoelectronics. Adv. Electron. Mater. 2016, 3, 1600529. [CrossRef]

4. Levy, D.; Castellon, E. Transparent Conductive Materials: Materials, Synthesis, Characterization, Applications, 1st ed.; Wiley-VCH Verlag Gmbh \&Co, KGaA: Weinheim, Germany, 2018.

5. Transparent Conductive Films Market by Application (Smartphones, Tablets, Notebooks, LCDs, Wearable Devices), Material (ITO on Glass, ITO on PET, Metal Mesh, Silver Nanowires, Carbon Nanotubes), and Region-Global Forecast to 2026. Available online: https://www.marketsandmarkets.com/Market-Reports/ transparent-conductive-film-market-59909084.html (accessed on 30 April 2020).

6. Suzuki, A.; Matsushita, T.; Wada, N.; Sakamoto, Y.; Okuda, M. Transparent conducting Al-doped ZnO thin films prepared by pulsed laser deposition. Jpn. J. Appl. Phys. 1996, 35, L56-L59. [CrossRef]

7. Berginski, M.; Hupkes, J.; Schulte, M.; Schope, G.; Stiebig, H.; Rech, B.; Wuttig, M. The effect of front ZnO:Al surface texture and optical transparency on efficient light trapping in silicon thin-film solar cells. J. Appl. Phys. 2007, 101, 074903. [CrossRef]

8. Minami, T.; Sato, H.; Nanto, H.; Takata, S. Heat treatment in hydrogen gas and plasma for transparent conducting oxide films such as $\mathrm{ZnO}, \mathrm{SnO}_{2}$ and indium tin oxide. Thin Solid Films 1989, 176, $277-282$. [CrossRef]

9. Dhere, R.G.; Bonnet-Eymard, M.; Charlet, E.; Peter, E.; Duenow, J.N.; Li, J.V.; Kuciauskas, D.; Gessert, T.A. CdTe solar cell with industrial Al:ZnO on soda-lime glass. Thin Solid Films 2011, 519, 7142-7145. [CrossRef]

10. Vasekar, P.S.; Dhere, N.G.; Moutinho, H. Development of $\mathrm{CIGS}_{2}$ solar cells with lower absorber thickness. Sol. Energy 2009, 83, 1566-1570. [CrossRef]

11. Park, S.; Ikegami, T.; Ebihara, K. Growth of transparent conductive Al-doped ZnO thin films and device applications. Jpn. J. Appl. Phys. 2006, 45, 8453-8456. [CrossRef]

12. Verma, A.; Khan, F.; Kumar, D.; Kar, M.; Chakravarty, B.C.; Singh, S.N.; Husain, M. Sol-gel derived aluminum doped zinc oxide for application as anti-reflection coating in terrestrial silicon solar cells. Thin Solid Films 2010, 518, 2649-2653. [CrossRef]

13. Mondragon-Suarez,H.; Maldonado, A.; de la L Olvera, M.; Reyes, A.; Castanedo-Perez, R.; Torres-Delgado, G.; Asomoza, R. ZnO:Al thin films obtained by chemical spray: Effect of the Al concentration. Appl. Surf. Sci. 2002, 193, 52-59. [CrossRef] 
14. Ma, J.; Ji, F.; Ma, H.; Li, S. Preparation and properties of transparent conducting zinc oxide and aluminium-doped zinc oxide films prepared by evaporating method. Sol. Energy Mater. Sol. Cells 2000, 60, 341-348. [CrossRef]

15. Liu, Y.; Lian, J. Optical and electrical properties of aluminum doped $\mathrm{ZnO}$ thin films grown by pulsed laser deposition. Appl. Surf. Sci. 2007, 253, 3727-3730. [CrossRef]

16. Yang, W.; Wu, Z.; Liu, Z.; Pang, A.; Tu, Y.; Feng, Z.C. Room temperature deposition of Al-doped ZnO films on quartz substrates by radio-frequency magnetron sputtering and effects of thermal annealing. Thin Solid Films 2010, 519, 31-36. [CrossRef]

17. Kon, M.; Song, P.K.; Shigesato, Y.; Frach, P.; Mizukami, A.; Suzuki, K. Al-doped ZnO films deposited by reactive magnetron sputtering in mid-frequency mode with dual cathodes. Jpn. J. Appl. Phys. 2002, 41, 814-819. [CrossRef]

18. Tynell, T.; Yamauchi, H.; Karppinen, M.; Okazaki, R.; Terasaki, I. Atomic layer deposition of Al-doped ZnO thin films. J. Vac. Sci. Technol. A 2013, 31, 01A109. [CrossRef]

19. Johnson, R.W.; Hultqvist, A.; Bent, S.F. A brief review of atomic layer deposition: From fundamentals to applications. Mater. Today 2014, 17, 236-246. [CrossRef]

20. Dhakal, T.; Nandur, A.S.; Christian, R.; Vasekar, P.; Desu, S.; Westgate, C.; Koukis, D.I.; Arenas, D.J.; Tanner, D.B. Transmittance from visible to mid infra-red in AZO films grown by atomic layer deposition system. Sol. Energy 2012, 86, 1306-1312. [CrossRef]

21. Tulzo, H.L.; Schneider, N.; Lincot, D.; Patriarche, G.; Donsanti, F. Impact of the sequence of precursor introduction on the growth and properties of atomic layer deposited Al-doped ZnO films. J. Vac. Sci. Technol. A 2018, 36, 041502. [CrossRef]

22. Blagoev, B.S.; Dimitrov, D.Z.; Mehandzhiev, V.B.; Kovacheva, D.; Terziyska, P.; Pavlic, J.; Lovchinov, K.; Mateev, E.; Leclercq, J.; Sveshtarov, P. Electron transport in Al-doped ZnO nanolayers obtained by atomic layer deposition. J. Phys. Conf. Ser. 2016, 700, 012040. [CrossRef]

23. Sweet, W.J., III; Jur, J.S.; Parsons, G.N. Bi-layer $\mathrm{Al}_{2} \mathrm{O}_{3} / \mathrm{ZnO}$ atomic layer deposition for controllable conductive coatings on polypropylene nonwoven fiber mats. J. Appl. Phys. 2013, 113, 194303. [CrossRef]

24. Yeh, P.; Gu, C. Optics of Liquid Crystal Display; Wiley Interscience: New York, NY, USA, 2010.

25. Khoo, I.C. Liquid Crystals Physical Properties and Nonlinear Optical Phenomena; Wiley: Hoboken, NJ, USA, 1995.

26. Haacke, G. New figure of merit for transparent conductors. J. Appl. Phys. 1976, 47, 4086-4089. [CrossRef]

27. Oh, B.-Y.; Jeong, M.-C.; Moon, T.-H.; Lee, W.; Myoung, J.-M.; Hwang, J.-Y.; Seo, D.-S. Transparent conductive Al-doped ZnO films for liquid crystal displays. J. Appl. Phys. 2006, 99, 124505. [CrossRef]

28. Su, Y.C.; Chiou, C.C.; Marinova, V.; Lin, S.H.; Bozhinov, N.; Blagoev, B.; Babeva, T.; Hsu, K.Y.; Dimitrov, D.Z. Atomic layer deposition prepared Al-doped $\mathrm{ZnO}$ for liquid crystal displays applications. Opt. Quantum Electron. 2018, 50, 205. [CrossRef]

29. Baetens, R.; Jelle, B.P.; Gustavsen, A. Properties, requirements and possibilities of smart windows for dynamic daylight and solar energy control in buildings: State-of-the-art. Sol. Energy Mater. Sol. Cells 2010, 94, 8-105. [CrossRef]

30. Chung, S.H.; Noh, H.Y. Polymer-dispersed liquid crystal devices with graphene electrodes. Opt. Express 2015, 23, 32149. [CrossRef]

31. Sannicolo, T.; Lagrange, M.; Cabos, A.; Celle, C.; Simonato, J.P.; Bellet, D. Metallic Nanowire-based transparent electrodes for next generation flexible devices: A review. Small 2016, 12, 6052-6075. [CrossRef]

32. Huang, Q.; Shen, W.; Fang, X.Z.; Chen, G.; Yang, Y.; Huang, J.; Tan, R.; Song, W. Highly thermostable, flexible, transparent, and conductive films on polyimide substrate with an AZO/AgNW/AZO structure. ACS Appl. Mater. Interfaces 2015, 7, 4299-4305. [CrossRef]

(C) 2020 by the authors. Licensee MDPI, Basel, Switzerland. This article is an open access article distributed under the terms and conditions of the Creative Commons Attribution (CC BY) license (http://creativecommons.org/licenses/by/4.0/). 